\title{
A Model for Digital Topology
}

\author{
Gilles Bertrand and Michel Couprie \\ Laboratoire A ${ }^{2}$ SI, ESIEE Cité Descartes B.P. 99 \\ 93162 Noisy-Le-Grand Cedex France, \\ e-mail: bertrang@esiee.fr, coupriem@esiee.fr
}

\begin{abstract}
In the framework known as digital topology, two different adjacency relations are used for structuring the discrete space $\mathcal{Z}^{n}$. In this paper, we propose a model for digital topology based on the notion of order and discrete topology. We "validate" our model by considering the two fundamental notions of surface and simple point. At last, we give the different possible configurations that may appear in 2- and 3dimensional surfaces in $\mathcal{Z}^{4}$ which correspond to our model.
\end{abstract}

Keywords: discrete topology, digital topology, surface, simple point

\section{Introduction}

Perhaps the simplest way for structuring the discrete space $\mathcal{Z}^{n}$ is to consider two graphs, these graphs correspond to two adjacency relations between the elements of $\mathcal{Z}^{n}$ : the direct and the indirect adjacency (for example, the 4 - and the 8-adjacency in $\mathcal{Z}^{2}$ ). This approach, known as digital topology [2212], is widely used in $\mathcal{Z}^{2}$ for the applications to image analysis. Nevertheless, severe limitations appear in the discrete space $\mathcal{Z}^{3}$. For example, in the pioneering work of Morgenthaler and Rosenfeld [19], a definition of surfaces in $\mathcal{Z}^{3}$ is proposed, using such adjacency relations. This definition is not based on structural properties which are fundamental for surfaces (e.g. the neighborhood of each point should constitute a "cycle" [8]). Hence, it is difficult to appreciate the relevancy of this definition. Furthermore, this definition seems to be almost impossible to generalize to higher dimensions.

In this paper, we consider an approach based on the notion of order and discrete topology $1,10,14,16$. We propose a model for digital topology by associating, to each subset of $\mathcal{Z}^{n}$, two orders: the hit (resp. miss) order corresponds to the indirect (resp. direct) adjacency.

We "validate" our model by considering the two fundamental notions of surface and simple point.

We show that the notion of surface in these two orders corresponds exactly to the notion of surface introduced by Morgenthaler and Rosenfeld. We also verify that the notion of simple point introduced in these two orders agrees with the corresponding notion in digital topology.

These results are remarkable, since the definitions of surface and simple point in the digital topology framework are fundamentaly different from ours. These 
differences explain why we used a computer in order to prove these results by an exhaustive checking. A hand-made proof would be of low interest.

At last, we give the different possible configurations that may appear in 2and 3-dimensional surfaces in $\mathcal{Z}^{4}$ which correspond to our model.

\section{Basic notions}

In this section, we introduce some basic notions relative to orders (see also 6]).

If $X$ is a set, $\mathcal{P}(X)$ denotes the set composed of all subsets of $X$, if $S$ is a subset of $X, \bar{S}$ denotes the complement of $S$ in $X$. If $S$ is a subset of $T$, we write $S \subseteq T$, the notation $S \subset T$ means $S \subseteq T$ and $S \neq T$. If $\gamma$ is a map from $\mathcal{P}(X)$ to $\mathcal{P}(X)$, the dual of $\gamma$ is the map $* \gamma$ from $\mathcal{P}(X)$ to $\mathcal{P}(X)$ such that, for each $S \subseteq X, * \gamma(S)=\overline{\gamma(\bar{S})}$. Let $\delta$ be a binary relation on $X$, i.e., a subset of $X \times X$. We also denote $\delta$ the map from $X$ to $\mathcal{P}(X)$ such that, for each $x$ of $X, \delta(x)=\{y \in X,(x, y) \in \delta\}$. We define $\delta^{\square}$ as the binary relation $\delta^{\square}=\delta \backslash\{(x, x) ; x \in X\}$.

An order is a pair $|X|=(X, \alpha)$ where $X$ is a set and $\alpha$ is a reflexive, antisymmetric, and transitive binary relation on $X$. An element of $X$ is also called a point. The set $\alpha(x)$ is called the $\alpha$-adherence of $x$, if $y \in \alpha(x)$ we say that $y$ is $\alpha$-adherent to $x$.

Let $(X, \alpha)$ be an order. We denote $\alpha$ the map from $\mathcal{P}(X)$ to $\mathcal{P}(X)$ such that, for each subset $S$ of $X, \alpha(S)=\cup\{\alpha(x) ; x \in S\}, \alpha(S)$ is called the $\alpha$-closure of $S, * \alpha(S)$ is called the $\alpha$-interior of $S$. A subset $S$ of $X$ is $\alpha$-closed if $S=\alpha(S)$, $S$ is $\alpha$-open if $S=* \alpha(S)$.

Let $(X, \alpha)$ be an order. We denote $\beta$ the relation $\beta=\{(x, y) ;(y, x) \in \alpha\}, \beta$ is the inverse of the relation $\alpha$. We denote $\theta=\alpha \cup \beta$. The dual of the order $(X, \alpha)$ is the order $(X, \beta)$.

Note that $* \alpha(S)=\{x \in S ; \beta(x) \subseteq S\}$, and $* \beta(S)=\{x \in S ; \alpha(x) \subseteq S\}$.

The set $\mathcal{O}_{\alpha}$ composed of all $\alpha$-open subsets of $X$ satisfies the conditions for the family of open subsets of a topology, the same result holds for the set $\mathcal{O}_{\beta}$ composed of all $\beta$-open subsets of $X$; we denote respectively $\mathcal{T}_{\alpha}=\left(X, \mathcal{O}_{\alpha}\right)$ and $\mathcal{T}_{\beta}=\left(X, \mathcal{O}_{\beta}\right)$ these two topologies. These topologies are Alexandroff topologies, i.e., topologies such that every intersection of open sets is open [1].

An order $(X, \alpha)$ is countable if $X$ is countable, it is locally finite if, for each $x \in X, \theta(x)$ is a finite set. A $C F$-order is a countable locally finite order.

Let $(X, \alpha)$ be a CF-order. Let $x_{0}$ and $x_{k}$ be two points of $X$. A path from $x_{0}$ to $x_{k}$ is a sequence $x_{0}, x_{1}, \ldots, x_{k}$ of elements of $X$ such that $x_{i} \in \theta\left(x_{i-1}\right)$, with $i=1, \ldots, k$.

It may be seen that a CF-order $(X, \alpha)$ is connected for $\mathcal{T}_{\alpha}$ (or for $\mathcal{T}_{\beta}$ ) if and only if it is path-connected, i.e, if for all $x, y$ in $X$, there is a path from $x$ to $y$.

When $(X, \alpha)$ and $\left(X^{\prime}, \alpha^{\prime}\right)$ are orders, a map $f$ from $X$ to $X^{\prime}$ is order preserving, or isotone, if $y \in \alpha(x)$ implies $f(y) \in \alpha^{\prime}[f(x)]$. We say that $f$ is an isomorphism from $(X, \alpha)$ to $\left(X^{\prime}, \alpha^{\prime}\right)$, if $f$ is a bijection so that $y \in \alpha(y)$ if and only if $f(y) \in \alpha^{\prime}[f(x)]$, for all $x, y$ in $X$. 
If $(X, \alpha)$ is an order and $S$ is a subset of $X$, the order relative to $S$ is the order $|S|=(S, \alpha \cap(S \times S))$.

Let $(X, \alpha)$ be an order. An element $x$ such that $\alpha^{\square}(x)=\emptyset$ is said to be $\alpha$-terminal (for $X$ ). A point $y$ is an $\alpha$-terminal of $x$ if $y$ is an $\alpha$-terminal and $y \in \alpha(x)$.

If $(X, \alpha)$ is an order, we define $\alpha^{\bullet}$ as the relation on $X$ such that $y \in \alpha^{\bullet}(x)$ if and only if $y \in \alpha^{\square}(x)$ and $\alpha^{\square}(x) \cap \beta^{\square}(y)=\emptyset$. The set $\alpha^{\bullet}(x)$ is called the $\alpha$-closeness of $x$, if $y \in \alpha^{\bullet}(x)$ we say that $y$ is $\alpha$-close to $x$.

Let $(X, \alpha)$ be a CF-order and let $x$ be a point of $X$. We say that $x$ is $\alpha$ unipolar if $\alpha^{\bullet}(x)$ consists in exactly one point. We say that $x$ is $\alpha$-free if there is a sequence $x_{0}, \ldots, x_{k}$ with $x_{k}=x$, such that $x_{0}$ is $\alpha$-unipolar and $x_{i}$ is $\alpha$-unipolar for the order $\left|X \backslash\left\{x_{0}, \ldots, x_{i-1}\right\}\right|, i=1, \ldots, k$. A point which is not $\alpha$-free is called an $\alpha$-link. The $\alpha$-kernel of $X$ is the subset of $X$ composed of all $\alpha$-links of $X$. Two orders $(X, \alpha)$ and $\left(X, \alpha^{\prime}\right)$ are said to be $\alpha$-equivalent if the orders induced by their $\alpha$-kernel are isomorphic.

We use a general definition for $n$-dimensional surfaces which has been proposed by Evako, Kopperman and Mukhin [7]. This notion is close to the notion of manifold used by Kovalevsky [17]; nevertheless it does not involve the necessity to attach a notion of dimension to each element of $X$, which allows to have a simpler definition (in particular, no use of isomorphism is made).

Definition 1: Let $|X|=(X, \alpha)$ be a non-empty CF-order.

- The order $|X|$ is a 0 -surface if $X$ is composed exactly of two points $x$ and $y$ such that $y \notin \alpha(x)$ and $x \notin \alpha(y)$.

- The order $|X|$ is an n-surface, $n>0$, if $|X|$ is connected and if, for each $x$ in $X$, the order $\left|\theta^{\square}(x)\right|$ is an $(n-1)$-surface.

- $A$ (closed) curve is a 1-surface, a (closed) surface is a 2-surface.

We will use the following notion of simple point as a model for the classical corresponding notion in $\mathcal{Z}^{2}$ and $\mathcal{Z}^{3}$ :

Definition 2: Let $|X|=(X, \alpha)$ be a non-empty CF-order.

- The order $|X|$ is 0-contractible if $X$ is composed of a single point.

- A point $x$ is $\alpha_{n}$-simple if $\left|\alpha^{\square}(x)\right|$ is $(n-1)$-contractible, $n>0$.

We denote $X_{\alpha n}$ the set composed of all points of $X$ which are not $\alpha_{n}$-simple points for $|X|$.

- The order $|X|$ is $n$-contractible, $n>0$, if there is a sequence $X^{0}, \ldots, X^{k}$ with $X^{0}=X$ and $X^{k}=\{a\}, a \in X$, such that $X^{i}=X_{\alpha n}^{i-1}$ if $i$ is odd, and $X^{i}=X_{\beta n}^{i-1}$ if $i$ is even, for $i=1, \ldots, k$.

\section{Orders associated to $\mathcal{Z}^{n}$}

We give now a presentation of some orders which may be associated to $\mathcal{Z}^{n}$.

Let $E$ be a set and let $E^{n}$ be the Cartesian product of $n$ copies of $E$. An element $a$ of $E^{n}$ may be seen as a map from $\{1, \ldots, n\}$ to $E, a(i)$ is the $i$-th 
coordinate of $a, i=1, \ldots, n$. If $S$ is a subset of $E^{n}$, the $i$-th projection of $S$ is the set $S(i)=\{a(i) ; a \in S\}, i=1, \ldots, n$.

Let $\mathcal{Z}$ be the set of relative integers. We consider the families of sets $H_{0}^{1}, H_{1}^{1}$, $H^{1}$ such that, $H_{0}^{1}=\{\{a\} ; a \in \mathcal{Z}\}, H_{1}^{1}=\{\{a, a+1\} ; a \in \mathcal{Z}\}, H^{1}=H_{0}^{1} \cup H_{1}^{1}$. A subset $S$ of $\mathcal{Z}^{n}$ which is the Cartesian product of exactly $m$ elements of $H_{1}^{1}$ and $(n-m)$ elements of $H_{0}^{1}$ is called a $m$-cube of $\mathcal{Z}^{n}$. We denote $H^{n}$ the set composed of all $m$-cubes of $\mathcal{Z}^{n}, m=0, \ldots, n$.

An $m$-cube of $\mathcal{Z}^{n}$ is called a singleton if $m=0$, a unit interval if $m=1$, a unit square if $m=2$, a unit cube if $m=3$.

In this paper, the basic order associated to $\mathcal{Z}^{n}$ is the order $\left(H^{n}, \alpha\right)$, where $\alpha=\supseteq$, thus $y \in \alpha(x)$ if $x \supseteq y$. In Fig. 1, an example of a subset $S$ of $H^{2}$ is given. The object $S$ is made of two connected components $S_{1}$ and $S_{2}$. It may be seen that $S_{1}$ contains one $\alpha$-terminal and four $\alpha$-free elements (three $\alpha$-unipolar elements and one element which is an $\alpha$-unipolar element after the removal of these three elements). It follows that the $\alpha$-kernel of $S_{1}$ is composed of a single element: $\left|S_{1}\right|$ is $\alpha$-equivalent to an isolated point. In a similar way $\left|S_{2}\right|$ is $\alpha$ equivalent to an open curve made of three elements.

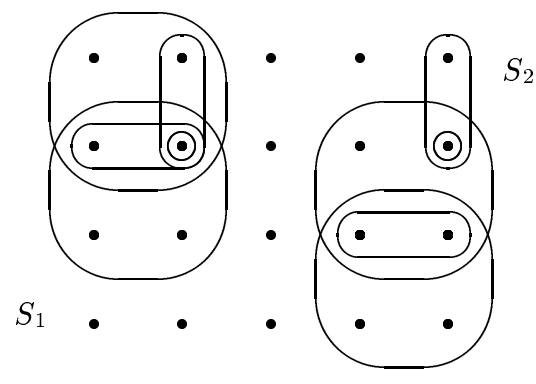

(a)

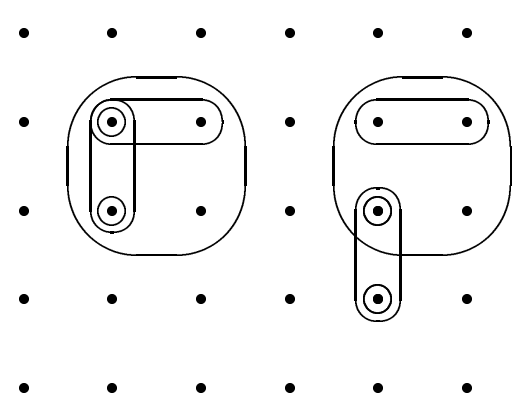

(b)

Fig. 1. A subset $S$ of $H^{2}$ (a), and its dual in $* H^{2}$ (b).

In the framework of 2D-image analysis, an element of an object is called a pixel. In a square grid, a pixel is sometimes seen as an element of $\mathcal{Z}^{2}$ and sometimes as an elementary square of $\mathcal{R}^{2}$. In fact, there is an equivalence between the two approaches. This comes from the fact that there is a "geometrical" duality in a square grid: if we consider the four elementary squares which contain an element of $\mathcal{Z}^{2}$, these four squares may be considered in turn as "constituting a unit square". Note that we do not have such a duality in an hexagonal grid. In order to make more explicit this kind of duality, we propose the following construction. 
We define the dual of $\mathcal{Z}$ as the set $* \mathcal{Z}=\{p+1 / 2 ; p \in \mathcal{Z}\}$. The dual of $\mathcal{Z}^{n}$ is the set $* \mathcal{Z}^{n}=[* \mathcal{Z}]^{n}$. We consider the families of sets $* H_{0}^{1}, * H_{1}^{1}, * H^{1}$ such that, $* H_{0}^{1}=\{\{a\} ; a \in * \mathcal{Z}\}, * H_{1}^{1}=\{\{a, a+1\} ; a \in * \mathcal{Z}\}, * H^{1}=* H_{0}^{1} \cup * H_{1}^{1}$.

A subset $S$ of $* \mathcal{Z}^{n}$ which is the Cartesian product of exactly $m$ elements of $* H_{1}^{1}$ and $(n-m)$ elements of $* H_{0}^{1}$ is called a $m$-cube of $* \mathcal{Z}^{n}$, we denote $* H^{n}$ the set composed of all $m$-cubes of $* \mathcal{Z}^{n}, m=0, \ldots, n$.

We denote $f$, the map from $H^{n}$ to $* H^{n}$, such that, for each $x$ of $H^{n}$, if $x(i)=$ $\{p, p+1\}$, then $[f(x)](i)=\{p+1 / 2\}$, and if $x(i)=\{p\}$, then $[f(x)](i)=$ $\{p-1 / 2, p+1 / 2\}, i=1, \ldots, n$. The dual of a $m$-cube of $H^{n}$ is the image under $f$ of this $m$-cube, it may be seen that the image of a $m$-cube is a $(n-m)$-cube. The dual of a subset $S$ of $H^{n}$ is the subset of $* H^{n}$ composed of the duals of all $m$-cubes in $S$. It may be seen that the map $f$ is an isomorphism between the two orders $\left(H^{n}, \supseteq\right)$ and $\left(* H^{n}, \subseteq\right)$. In Fig. 1 , the dual of a subset $S$ of $H^{2}$ is represented.

To conclude this section, we introduce some adjacency relations among elements of $\mathcal{Z}^{n}$ which may be naturally derived from the order $H^{n}$.

Two elements $p$ and $q$ of $\mathcal{Z}^{n}$ are said to be $m$-adjacent if there exists a $m$-cube of $H^{n}$ which contains both $x$ and $y$. Two elements of $\mathcal{Z}^{n}$ are strictly 0-adjacent if they are 0 -adjacent (i.e. if they are equal), they are strictly m-adjacent if they are $m$-adjacent and not $(m-1)$-adjacent, with $m=1, \ldots, n$.

\section{The $i / d$ adjacency approach}

In this section we give formal notions which correspond to the graph-theoretic approach introduced by Rosenfeld, this approach is based on two adjacencies relations, the $i$ - and $d$-adjacencies.

Two elements $p$ and $q$ of $\mathcal{Z}^{n}$ are said to be indirectly adjacent or $i$-adjacent (resp. directly adjacent or $d$-adjacent) if they are $n$-adjacent (resp. 1 -adjacent). It is well known that, in order to avoid connectivity paradoxes, we never use the same adjacency on both $S$ and $\bar{S}$. If we use the $i$-adjacency (resp. $d$-adjacency) for $S$, we must use the $d$-adjacency (resp. $i$-adjacency) for $\bar{S}$. In the sequel the $a$-adjacency indicates the $i$ - or the $d$-adjacency, i.e., we have $a=i$ or $a=d$.

Let $p$ be an element of $\mathcal{Z}^{n}$. We denote $\Gamma_{a}(p)$ the set composed of all elements of $\mathcal{Z}^{n}$ which are $a$-adjacent to $p$. The a-neighborhood of $p$ is the set $\Gamma_{a}^{*}(p)=$ $\Gamma_{a}(p) \backslash\{p\}$. An element $q$ of $\Gamma_{a}^{*}(p)$ is called a a-neighbor of $p$.

For example, each $p \in \mathcal{Z}^{2}$ has $8 i$-neighbors and $4 d$-neighbors, each $p \in \mathcal{Z}^{3}$ has $26 i$-neighbors and $6 d$-neighbors.

An $a$-path is a sequence $p_{0}, \ldots, p_{k}, p_{i}$ being $a$-adjacent to $p_{i-1}, i=1, \ldots, k$.

A subset $S$ of $\mathcal{Z}^{n}$ is a-connected if for all $p, q$ of $S$ there is an $a$-path from $p$ to $q$ which is composed solely of elements of $S$.

A subset $S$ of $\mathcal{Z}^{n}$ is a simple closed a-curve if $S$ is a-connected and if the $a$ neighborhood of each element of $S$ is made of exactly two elements.

Let us consider the digital plane $\mathcal{Z}^{2}$. This set must be interpreted as a (nonbounded) surface. Thus it might be desirable that the neighborhood of each point of $\mathcal{Z}^{2}$ could be interpreted by something like a simple closed curve. We 
see that the $d$-neighborhood of a point is made of four elements, and that any couple of these elements are not $d$-adjacent. In this sense the $d$-neighborhood of a point is "under-connected". In a similar way, we see that the $i$-neighborhood of a point is "over-connected". These peculiarities explain many of the difficulties encountered when using the $i / d$ adjacency approach. Since the basic notion of a neighborhood is not appropriate, only ad-hoc definitions are possible. For example, the notion of surface in $\mathcal{Z}^{3}$ is far from being clear. Morgenthaler and Rosenfeld (MR) proposed the following definition (see [19], [20, 21], 11]).

Let $(a, \bar{a})=(i, d)$ or $(d, i)$.

A $M R$ a-surface is a finite connected subset $S$ of $\mathcal{Z}^{3}$ such that, for each $p \in S$, we have:

i) $\left[\Gamma_{i}^{*}(p) \cap S\right]$ has exactly one $a$-component $a$-adjacent to $p$; and

ii) $\left[\Gamma_{i}^{*}(p) \cap \bar{S}\right]$ has exactly two $\bar{a}$-components which are $\bar{a}$-adjacent to $\{p\}$, we denote $C$ and $D$ these components; and

iii) $\forall q \in\left[\Gamma_{a}^{*}(p) \cap S\right], q$ is $\bar{a}$-adjacent to both $C$ and $D$.

We conclude this section by giving a characterization of simple points in $\mathcal{Z}^{3}$ $(135)$.

Let $S \subset \mathcal{Z}^{3}$ and $p \in \mathcal{Z}^{3}$. Let $(a, \bar{a})=(i, d)$ or $(d, i)$.

We denote $\Gamma_{2}(p)=\left\{q \in \mathcal{Z}^{3}, q\right.$ is strictly 2 -adjacent to $\left.p\right\}$.

The geodesic a-neighborhood $G_{a}^{S}(p)$ of $p$ inside $S$ is defined by:

$G_{i}^{S}(p)=\Gamma_{i}^{*}(p) \cap S$

$G_{d}^{S}(p)=\left(\Gamma_{d}^{*}(p) \cap S\right) \cup\left\{q \in \Gamma_{2}(p) \cap S ; \operatorname{card}\left(\Gamma_{d}^{*}(p) \cap \Gamma_{d}^{*}(q) \cap S\right)=2\right\}$

A point $p \in \mathcal{Z}^{3}$ is a-simple (for $S$ ) if and only if the number of $a$-components in $G_{a}^{S}(p)$ and the number of $\bar{a}$-components in $G_{\bar{a}}^{S}(p)$ both equal one.

\section{The $h / m$ order approach}

In order to build consistent topological notions for a subset $S$ of $\mathcal{Z}^{n}$, we associate to $S$ a subset $\Psi(S)$ of $H^{n}$; thus we recover the structure of a (discrete) topological space by considering the order $\left(H^{n}, \supseteq\right)$. In this paper, the transformation $\Psi$ is chosen in such a way that the induced topological notions may be seen as a "model" for the notions derived from the $i / d$ adjacency framework. A natural idea for defining $\Psi$ is to consider "hit or miss" transformations [23]. Thus we consider the set $S^{h}$ composed of all elements of $H^{n}$ which have a non-empty intersection with $S$. In a dual way, we consider the set $S^{m}$ composed of all elements of $H^{n}$ which are included in $S$. Our model for the $i / d$ adjacency approach consists in considering the $\alpha$-kernels of the two sets $S^{h}$ and $S^{m}$ as counterparts for the $i$ - and $d$-adjacencies.

Definition 3: Let $S \subset \mathcal{Z}^{n}$. We denote $S^{h}=\left\{x \in H^{n} ; x \cap S \neq \emptyset\right\}$, and $S^{m}=\left\{x \in H^{n} ; x \subseteq S\right\}$. The h-set associated to $S$ is the set $\Psi^{h}(S)$ which is the $\alpha$-kernel of $S^{h}$. The $m$-set associated to $S$ is the set $\Psi^{m}(S)$ which is the $\alpha$-kernel of $S^{m}$. The h-order relative to $S$ is the order $\left(\Psi^{h}(S), \alpha\right)$, the $m$-order relative to $S$ is the order $\left(\Psi^{m}(S), \alpha\right)$, with $\alpha=\supseteq$. 


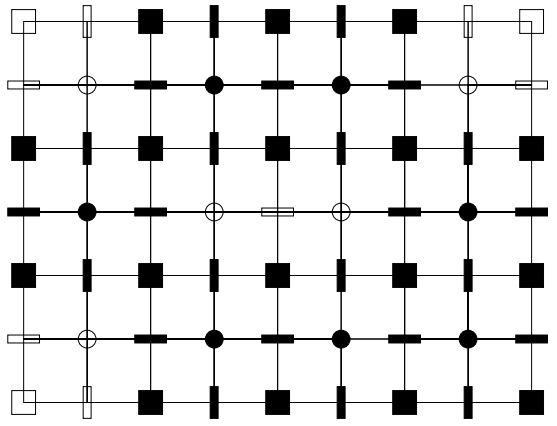

(a)

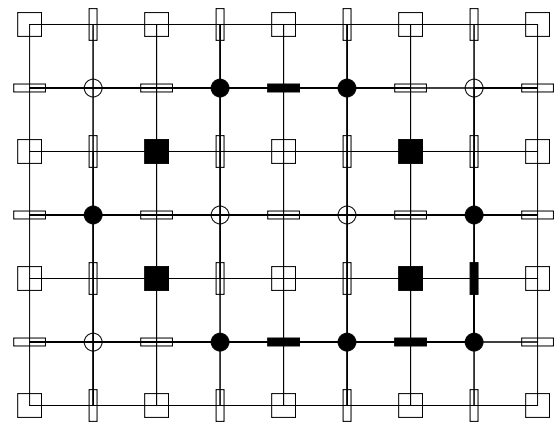

$(b)$

Fig. 2.(a): a subset $S$ of $\mathcal{Z}^{2}$ (the seven black dots) and the set $S^{h}$ (black elements); (b): the $h$-order relative to $S$.

We note that:

$-\overline{S^{m}}=[\bar{S}]^{h}$;

- The set $S^{h}$ is $\alpha$-open, the set $S^{m}$ is $\alpha$-closed;

- We have $\Psi^{m}(S)=S^{m}$;

- The set $\Psi^{h}(S)$ is, in general, neither $\alpha$-open nor $\alpha$-closed.

The proofs of the two following properties will be given in a forthcoming paper:

Property 4: Let $S \subset \mathcal{Z}^{n}$. The set $\Psi^{h}(S)$ is composed of all elements $\{p\}, p \in S$, and all $m$-cubes of $\mathcal{Z}^{n}$ which contain two strictly $m$-adjacent points $q$ and $q^{\prime}$ of $S, m=1, \ldots, n$.

Property 5: Let $S \subset \mathcal{Z}^{n}$. The dual of $\Psi^{h}(S)$ is composed of the duals of all elements $\{p\}, p \in S$, and all the $m$-cubes of $\mathcal{Z}^{n}$ which are the intersection of the dual of $\{q\}$ and the dual of $\left\{q^{\prime}\right\}$ for some $q, q^{\prime} \in S$.

In Fig. 2 (a), a set $S^{h}$ is represented. The original set $S$ is represented by the seven black dots. We use the following conventions: a singleton is depicted by a circle $(\circ)$, a unit interval by a rectangle $(\square)$, and a unit square by a square $(\square)$. Two elements $a, b$ are linked by a straight line if $a \in \alpha^{\bullet}(b)$ or $b \in \alpha^{\bullet}(a)$. In Fig. 2 (b), $\Psi^{h}(S)$, the $\alpha$-kernel of the set $S^{h}$, is given.

It may be easily seen that:

Property 6: Let $S \subset \mathcal{Z}^{n}$.

The set $S$ is a closed curve for the h-order (resp. $m$-order) if and only if $S$ is a closed $i$-curve (resp. closed d-curve).

The following property has been proven by an exhaustive checking with the help of a computer:

Property 7: Let $S \subset \mathcal{Z}^{3}$, let $x$ be a point of $S$.

The point $x$ is $i$-simple if and only if $\{x\}$ is $\alpha_{3}$-simple for the $h$-order. The point $x$ is d-simple if and only if $\{x\}$ is $\alpha_{3}$-simple for the $m$-order. 


\section{The $h$-surfaces and $m$-surfaces in $\mathcal{Z}^{3}$}

Let $S \subset \mathcal{Z}^{3}$, we say that $S$ is an $h$-surface (resp. an $m$-surface) if $S$ is a closed surface for the $h$-order (resp. $m$-order).

In this section, we show that $h$-surfaces (resp. $m$-surfaces) are equivalent to the Morgenthaler and Rosenfeld (MR) $i$-surfaces (resp. $d$-surfaces) [19|2021]. By construction, the MR's definition cannot be extended in higher dimensional spaces. Furthermore, it may be asked whether it is possible to recover the fondamental structure of a combinatorial 2D-manifold ([8]) where each element is "surrounded by a cycle". In fact an answer to this question has been given by Ayala and Al. (see [2, 3], 4]) in an original framework which provides a link between digital spaces and Euclidean spaces. A digital object is defined as a digital manifold if its continuous analogue is a combinatorial manifold. In this framework, it has been shown that Morgenthaler's surfaces are combinatorial surfaces. In a previous work, Kong and Roscoe [11] had proposed a structural necessary and sufficient condition based on specific point configurations, that characterizes the MR surfaces. Their approach is also based on continuous analogs.

On the opposite, our approach is purely discrete. By drawing the inferences of the definition of a 2 -surface, we recover very simply the local point configurations that may appear in $h$ - or $m$-surfaces. We can even exhibit in the next section, the different configurations that may appear in $h$-surfaces in $\mathcal{Z}^{4}$, as well as in 3 -surfaces for $h$-orders in $\mathcal{Z}^{4}$.

In the subsequent figures, we use the same conventions as for the twodimensional case, in addition, a unit cube is represented by a cube ( $\square)$.

The following property is a direct consequence of Def. 1:

Property 8: If $S \subset \mathcal{Z}^{3}$ is an m-surface, then each $\beta$-terminal element $x$ of $\Psi^{m}(S)$ is a unit square, and $\theta^{\square}(x)$ is a closed curve composed of four singletons and four unit intervals (see $b_{1}$, Fig 3).

Property 9: $A$ subset $S$ of $\mathcal{Z}^{3}$ is a $M R$ d-surface if and only if $S$ is an $m$ surface.

In the sequel, we will use "utrs" as an abbreviation for "up to rotations and symmetries".
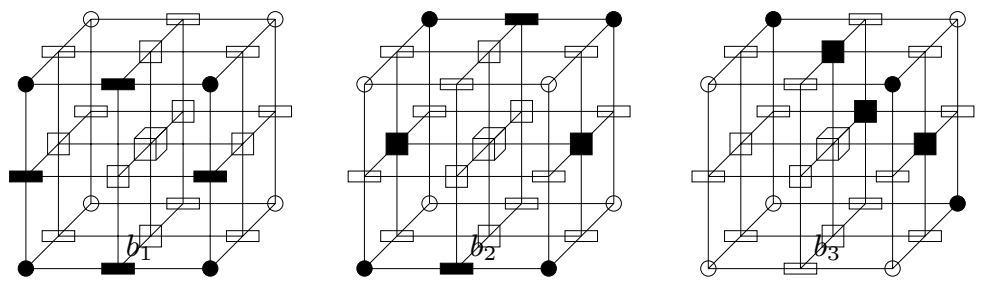

Fig. 3. The 3 possible configurations for the set $\theta^{\square}(x)$ of a $\beta$-terminal $x$ in an $h$-surface. The $\beta$-terminal $x$ is the front central unit square in $b_{1}$, the central unit cube in $b_{2}$ and $b_{3}$. 
Property 10: If $S \subset \mathcal{Z}^{3}$ is an h-surface, then

i) for each $\beta$-terminal element $x$ of $\Psi^{h}(S), \theta^{\square}(x)$ corresponds (utrs) to one of the 3 configurations $b_{i}(i=1 \ldots 3)$ depicted in Fig. 3, and

ii) for each $\alpha$-terminal element $x$ of $\Psi^{h}(S), \theta^{\square}(x)$ corresponds (utrs) to one of the 13 configurations $a_{i}(i=1 \ldots 13)$ depicted in Fig. 4.

The proof of this property is easily obtained by enumerating the different possible cases.

It may be seen that each of the configurations $a_{i}$ corresponds (utrs) to one of the configurations that may appear in a MR $i$-surface (see for example [3], Fig. 3). We have indeed:

Property 11: $A$ subset $S$ of $\mathcal{Z}^{3}$ is a $M R$ i-surface if and only if $S$ is an $h$ surface.
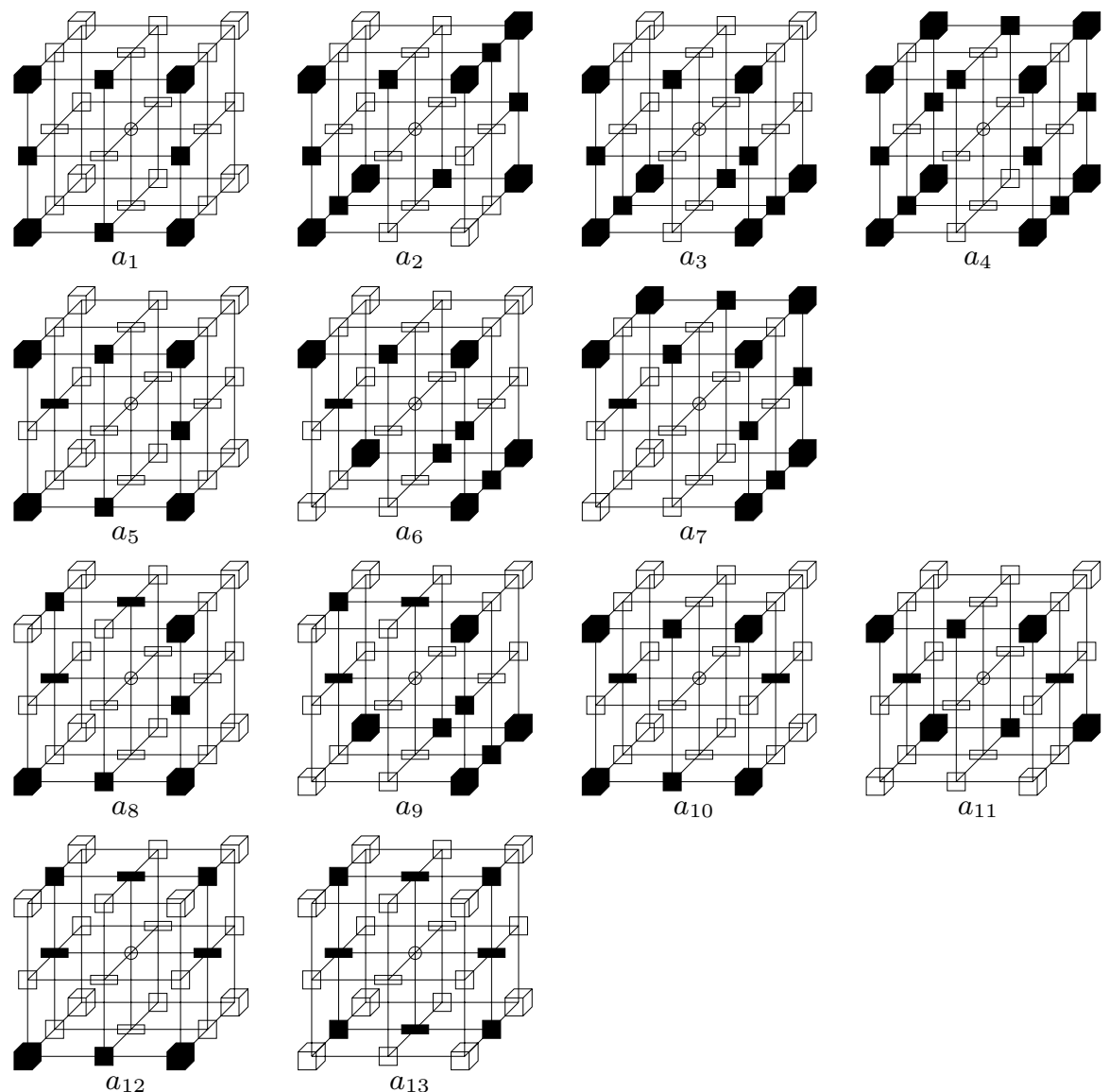

Fig. 4. The 13 possible configurations for the set $\theta^{\square}(x)$ of an $\alpha$-terminal $x$ in an $h$ surface. The $\alpha$-terminal $x$ is the central singleton. 


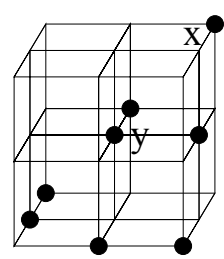

(a)

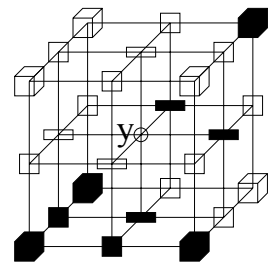

(b)

Fig. 5.(a): a configuration in which $y$ satisfies the MR's definition, but which may not appear in a MR $i$-surface; (b): the set $\theta^{\square}(y)$ for the $h$-order.

Remark: let us consider the configuration (a) in Fig. 5. We remark that $y$ is an $i$-surface point, according to MR's definition. But it is not possible that $x$ satisfies the conditions of the MR's definition, whatever neighborhood it may have; in other words, the configuration (a) is not extensible (see [18] for a precise definition of this notion).

With Def. 1, the configuration (a) is directly rejected from the list of configurations that may appear in an $h$-surface: $\theta^{\square}(y)$ is not a closed curve (see Fig. $5(\mathrm{~b}))$.

\section{The 2-surfaces and 3-surfaces for $h$-orders in $\mathcal{Z}^{4}$}

In the Fig. 6,7 , the set $\theta(x), x$ being a 4 -cube of $\mathcal{Z}^{4}$, is represented by three diagrams of 27 elements, $s_{1}, s_{2}, s_{3}$ (from left to right). For each of these three diagrams, we use the same conventions as for the figures of the previous section, concerning the inclusion relation of the order $\left(H^{4}, \supseteq\right)$. In addition, let us consider the two vectors $\vec{a}$ and $-\vec{a}$ in Fig. 6 : each element $x$ of $s_{1}$ is included in $x^{\prime}$, the translated of $x$ by $\vec{a}$, and each element $y$ of $s_{3}$ is included in $y^{\prime}$, the translated of $y$ by $-\vec{a}$. For example in Fig. $6\left(c_{4}\right)$, we have: $x \subset y, x \subset z, x \subset x^{\prime}, z \subset z^{\prime}, x^{\prime} \subset z^{\prime}$.

Property 12: If $S \subset \mathcal{Z}^{4}$ is an $h$-surface, then for each $\beta$-terminal element $x$ of $\Psi^{h}(S), \theta^{\square}(x)$ corresponds (utrs) to one of the 6 configurations $c_{i}(i=1 \ldots 6)$ : $c_{1}, c_{2}, c_{3}$ correspond respectively to $b_{1}, b_{2}, b_{3}$ depicted in Fig. 3, and $c_{4}, c_{5}, c_{6}$ are depicted in Fig. 6.

Property 13: If $S \subset \mathcal{Z}^{4}$ is a 3-surface for the $h$-order, then for each $\beta$-terminal element $x$ of $\Psi^{h}(S), \theta^{\square}(x)$ corresponds (utrs) to one of the 5 configurations $d_{i}$ ( $i=1 \ldots 5)$ depicted in Fig. 7 .

\section{References}

1. P. Alexandroff, "Diskrete Räume", Mat. Sbornik, 2, pp. 501-518, 1937.

2. R. Ayala, E. Domínguez, A.R. Francés, A. Quintero, J. Rubio, "On surfaces in digital topology", 5th Colloquium Discrete Geometry for Comp. Imagery, pp. 271$276,1995$. 
$c_{4}$

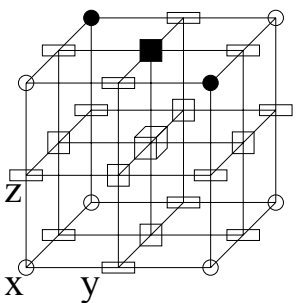

a

$c_{5}$
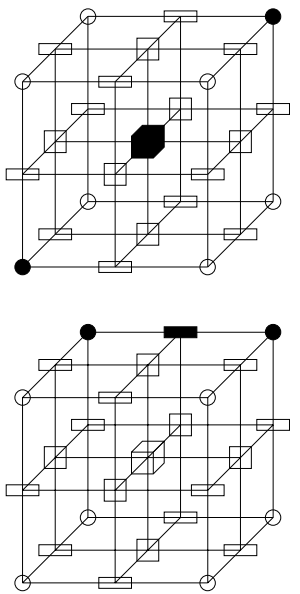
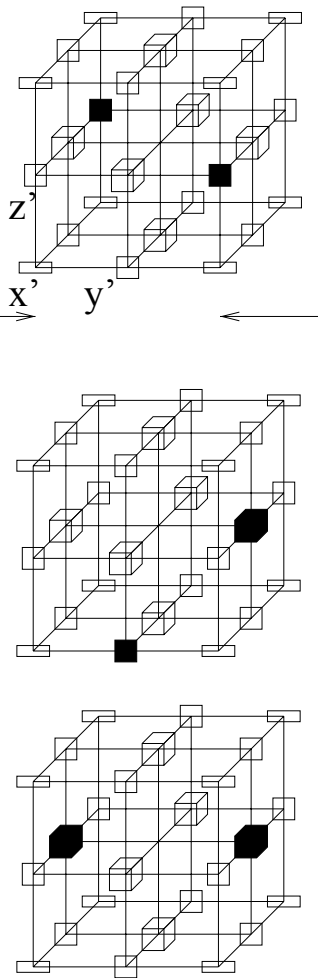
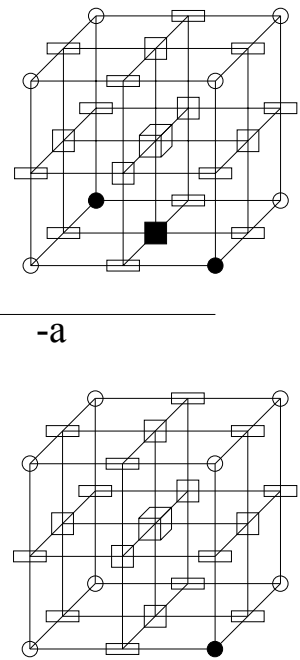

$-\mathrm{a}$

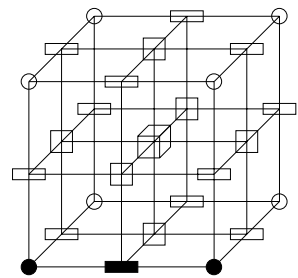

Fig. 6. Three of the six possible configurations for the set $\theta^{\square}(x)$ of a $\beta$-terminal $x$ in an $h$-surface in $\mathcal{Z}^{4}$ (the other three correspond to those in Fig. 3).

3. R. Ayala, E. Domínguez, A.R. Francés, A. Quintero, "Determining the components of the complement of a digital $(n-1)$-manifold in $\mathcal{Z}^{n}$, Discrete Geometry for Computer Imagery, Vol. 1176, Lect. Notes in Comp. Science, Springer Verlag, pp. 163-176, 1996.

4. R. Ayala, E. Domínguez, A.R. Francés, A. Quintero, "Digital lighting functions", Discrete Geometry for Computer Imagery, Vol. 1347, Lect. Notes in Comp. Science, Springer Verlag, pp. 139-150, 1997.

5. G. Bertrand, "Simple points, topological numbers and geodesic neighborhoods in cubic grids", Pattern Rec. Letters, Vol. 15, pp. 1003-1011, 1994.

6. G. Bertrand, "New notions for discrete topology", 8th Conf. on Discrete Geom. for Comp. Imag., Vol. 1568, Lect. Notes in Comp. Science, Springer Verlag, pp. 216-226, 1999.

7. A.V. Evako, R. Kopperman, Y.V. Mukhin, "Dimensional Properties of Graphs and Digital Spaces", Jour. of Math. Imaging and Vision, 6, pp. 109-119, 1996.

8. J. Françon, "Discrete combinatorial surfaces", CVGIP: Graphical Models and Image Processing, Vol. 57, pp. 20-26, 1995.

9. J. Françon, "On recent trends in discrete geometry in computer science", Conf. on Discrete Geometry for Comp. Imag., pp. 3-16, 1996. 

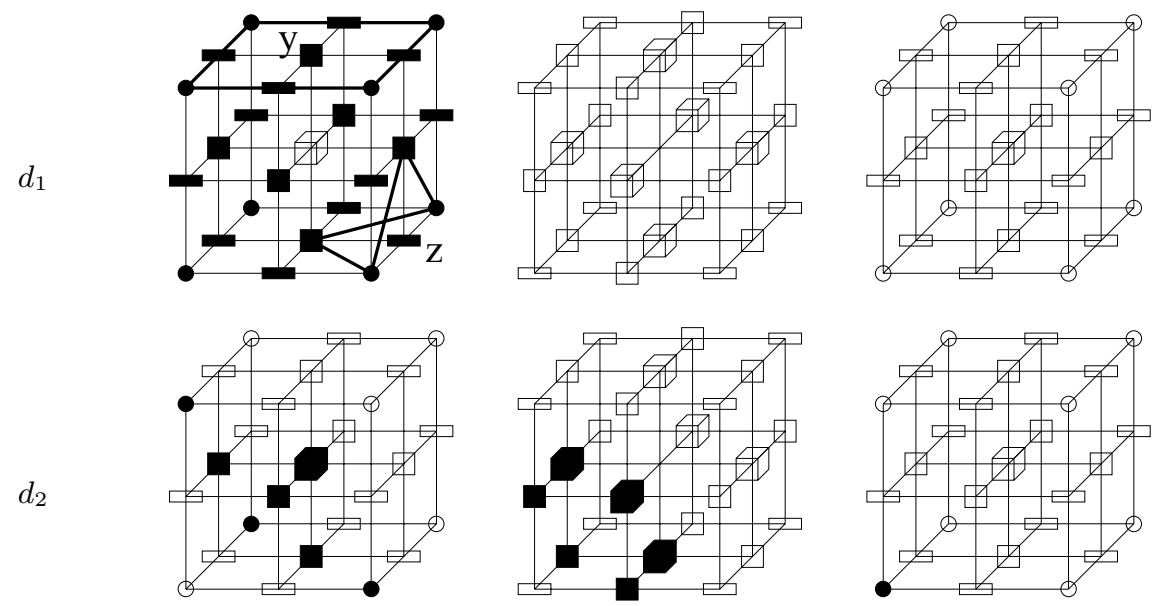

$d_{3}$
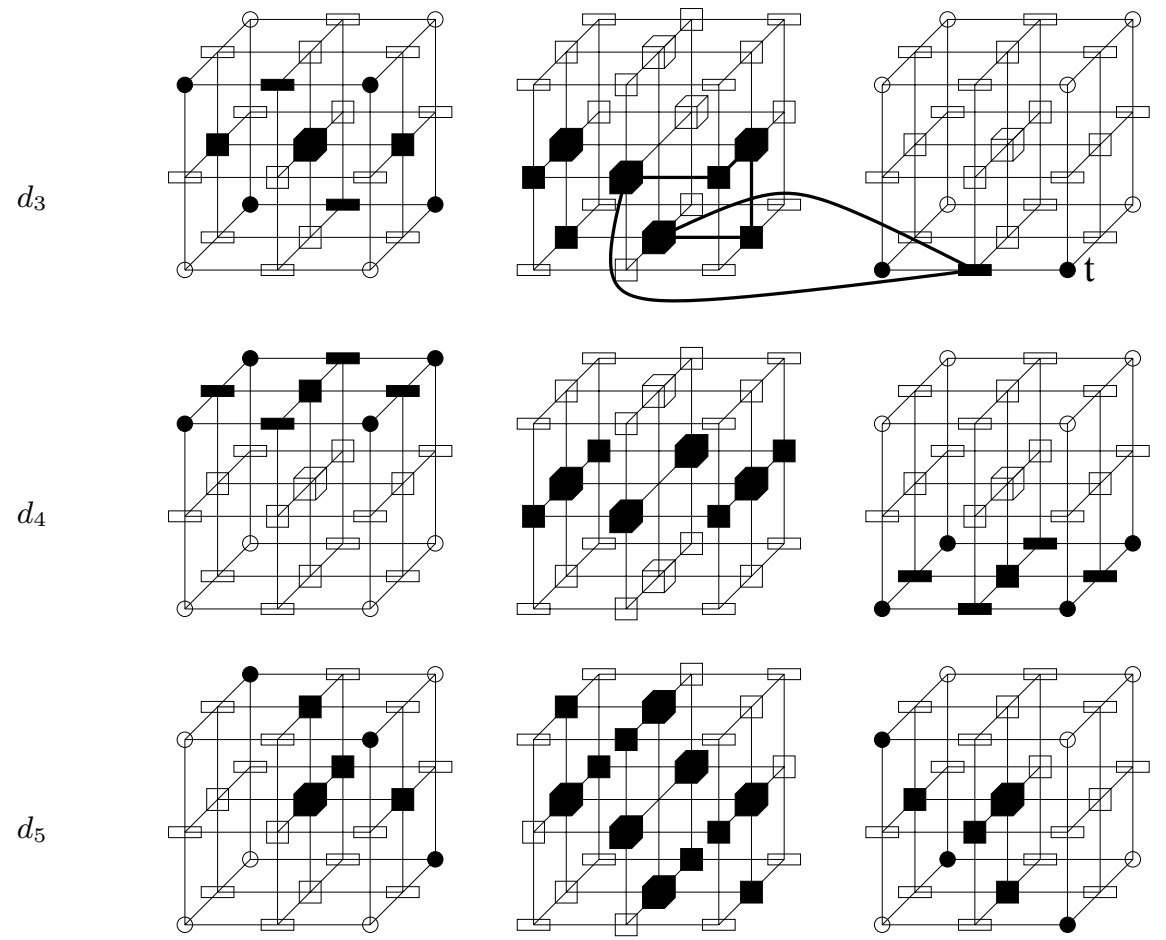

Fig. 7. The 5 possible configurations for the set $\theta^{\square}(x)$ of a $\beta$-terminal $x$ in a 3 -surface for $h$-orders in $\mathcal{Z}^{4}$. This set constitutes a 2 -surface (a closed surface). For three elements $p=y, z, t$, the set $\theta^{\square}(p)$ is represented with bold lines. This set constitutes a 1-surface (a closed curve). 
10. E. Khalimsky, R. Kopperman, P. R. Meyer, "Computer Graphics and Connected Topologies on Finite Ordered Sets", Topology and its Applications, 36, pp. 1-17, 1990.

11. T. Y. Kong, A. W. Roscoe, "Continuous Analogs of Axiomatized Digital Surfaces", Computer Vision, Graphics, and Image Processing, 29, pp. 60-86, 1985.

12. T.Y. Kong and A. Rosenfeld, "Digital Topology: introduction and survey", Comp. Vision, Graphics and Image Proc., 48, pp. 357-393, 1989.

13. T.Y. Kong, "Topology-Preserving Deletion of 1's from 2-, 3- and 4-Dimensional Binary Images", Conf. on Discrete Geometry for Comp. Imag., Lect. Notes in Comp. Science, Vol. 1347, Springer Verlag, pp. 3-18, 1997.

14. T. Y. Kong, R. Kopperman, P. R. Meyer, "A Topological Approach to Digital Topology", American Mathematical Monthly, 38, pp. 901-917, 1991.

15. R. Kopperman, P.R. Meyer and R.G. Wilson, "A Jordan surface theorem for threedimensional digital spaces", Discrete Comput. Geom., 6, pp. 155-161, 1991.

16. V. A. Kovalevsky, "Finite Topology as Applied to Image Analysis", Computer Vision, Graphics, and Image Processing, 46, pp. 141-161, 1989.

17. V.A. Kovalevsky, "Topological foundations of shape analysis", in Shape in Pictures, NATO ASI Series, Series F, Vol. 126, pp. 21-36, 1994.

18. R. Malgouyres, "A definition of surfaces of $Z^{3}$ ", Conf. on Discrete Geometry for Comp. Imag., pp. 23-34, 1993. See also Doctoral dissertation, Université d'Auvergne, France, 1994.

19. D.G. Morgenthaler and A. Rosenfeld, "Surfaces in three-dimensional images", Information and Control, 51, 227-247, 1981.

20. G.M. Reed and A. Rosenfeld, "Recognition of surfaces in three-dimensional digital images", Information and Control, Vol. 53, pp. 108-120, 1982.

21. G.M. Reed, "On the characterization of simple closed surfaces in three-dimensional digital images", Computer Vision, Graphics and Image Proc., Vol. 25, pp. 226-235, 1984 .

22. A. Rosenfeld, "Digital Topology", Amer. Math. Monthly, 86, pp. 621-630, 1979.

23. J. Serra, Image Analysis and Mathematical Morphology, Academic Press, 1982. 